\title{
Latest results for Proton-proton Cross Section Measurements with the TOTEM experiment at LHC.
}

\author{
Francesco S. Cafagna*, for the TOTEM Collaboration ${ }^{\dagger}$ \\ INFN Sezione di Bari, Italy \\ E-mail: francesco.cafagna@ba.infn.it
}

The precise knowledge of the proton-proton cross section is extremely important to model the development, in the atmosphere, of the showers induced by the interaction of ultra high energy cosmic rays.

The TOTEM (TOTal cross section, Elastic scattering and diffraction dissociation Measurement at the LHC) experiment, located at the interaction point 5 of the LHC, has measured the total, elastic and inelastic proton-proton cross-sections, using a luminosity independent method, based on the optical theorem, in a center-of-mass energy range from 2.76 to $13 \mathrm{TeV}$. The elastic scattering was investigated in a wide range of the squared four-momentum transfer $|t|$ allowing the study of Coulomb-nuclear interference region down to $|t| \sim 8 \times 10^{-4} \mathrm{GeV}^{2}$. This made possible the first measurement of the $\rho$ parameter at $\sqrt{s}=13 \mathrm{TeV}, \rho$ being the ratio between the real and the imaginary part of the nuclear elastic scattering amplitude at $t=0$. This measurement, combined with the total cross-section results, led to the exclusion of all the models classified and published by the COMPETE Collaboration. The results obtained by TOTEM are indeed compatible with predictions of a colorless 3-gluon bound state exchange in the t-channel of proton-proton elastic scattering, as postulated by alternative theoretical models both in the Regge-like framework and in the modern QCD framework.

In this contribution the latest TOTEM will be given, along with the actual experiment status and the future physics program for the LHC run 3 .

36th International Cosmic Ray Conference -ICRC2019-

July 24th - August 1st, 2019

Madison, WI, U.S.A.

\footnotetext{
* Speaker.

${ }^{\dagger}$ for collaboration list see PoS(ICRC2019)1177, see also http://cern.ch/totem
} 


\section{Introduction}

The LHC energy begins to overlap with the energy range where extreme energy cosmic-ray showers are studied. Investigations of proton-proton interactions at the LHC are therefore of high importance for the study of the development of cosmic-ray showers in the atmosphere and thus for the measurements of the high-energy cosmic-ray spectra and composition. One of the most important observable: the shower maximum $\left(\mathrm{X}_{\max }\right)$, strongly depends on the inelastic proton-air crosssection and calculations of hadron-nucleus cross-sections in the Glauber-Gribov formalism require the knowledge of the inelastic $p p$ cross-section along with the $p p$ elastic scattering amplitude in the small $|t|$ range. In addition, all kinds of diffractive phenomena influence the development of the shower cascade and the multiplicity fluctuations of secondary hadrons [1]. Thus, the measurements of the pp cross-sections and the particle flow are of high importance for the interpretation of cosmic ray showers.

TOTEM measured the total $p p$ cross-section at the energy of $\sqrt{s}=2.76,7,8$ and $13 \mathrm{TeV}$ $[14,2,3,11]$, using a luminosity, $\mathscr{L}$, independent method and validating it by comparing several methods to determine the total cross-sections $[4,5,6,7,12]$. The total cross-section can be obtained via the optical theorem that combines the total inelastic rate, $N_{\text {inel }}$, and the total nuclear elastic rate, $N_{e l}$, with its extrapolation to the optical point $t=0, \mathrm{~d} N_{e l} /\left.\mathrm{d} t\right|_{t=0}$ :

$$
\sigma_{\text {tot }}=\frac{16 \pi}{1+\rho^{2}} \frac{\left(\mathrm{d} N_{e l} / \mathrm{d} t\right)_{t=0}}{\left(N_{e l}+N_{\text {inel }}\right)}
$$

where $\rho$ is the ratio of the real to imaginary part of the hadronic scattering amplitude at $t=0$. The method requires the simultaneous measurements of the inelastic and elastic rates, as well as the extrapolation of the latter in the invisible region down to $|t|=0$. This is achieved with the experimental set-up of TOTEM which consists of two inelastic telescopes, called T1 and T2, to detect charged particles produced in inelastic $p p$ collisions, and Roman Pot stations to detect elastically scattered protons at very small angles. The measured ratio of the elastic and inelastic rates $N_{e l} / N_{\text {inel }}$ allows for the determination of the elastic and inelastic cross-sections as well.

The optical theorem can be used also to derive the total and fully inclusive inelastic $p p$ crosssection, from the measurement of the elastic scattering one:

$$
\sigma_{t o t}^{2}=\left.\frac{16 \pi}{1+\rho^{2}} \frac{\mathrm{d} \sigma_{e l}}{\mathrm{~d} t}\right|_{t=0}, \quad \sigma_{\text {inel }}=\sigma_{t o t}-\sigma_{e l} .
$$

To access to the smaller $|t|$-value region, the colliding beams must have a beam divergence of not more than a few micro-radians[8]. This can be obtained by either increasing the beta function value, $\beta^{*}$, or by reducing the beam emittance, $\varepsilon$ (beam divergence $=\sqrt{\varepsilon / \beta^{*}}$ ). With special runs having dedicated beam optics configurations, $\beta^{*}=90,1000,2500 \mathrm{~m}$, TOTEM extended the measurement to $|t|$-values as low as $8 \times 10^{-4} \mathrm{GeV}^{2}$, measuring the differential elastic cross-section over a wide range of $t[7,4,9,12]$. This made the extrapolation of the differential cross-section to the optical point at $t=0$ possible, allowing, for the first time at the LHC, the determination of the elastic scattering cross-section, the total cross-section via the optical theorem as well as the measurement of the $\rho$ parameter [12].

To increase robustness and understanding of the systematic uncertainties, the TOTEM Collaboration, cross checked the results of its measurements by comparing several independent ap- 


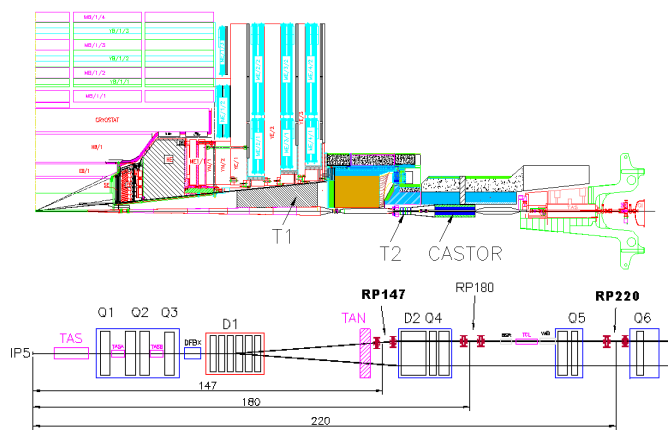

a)

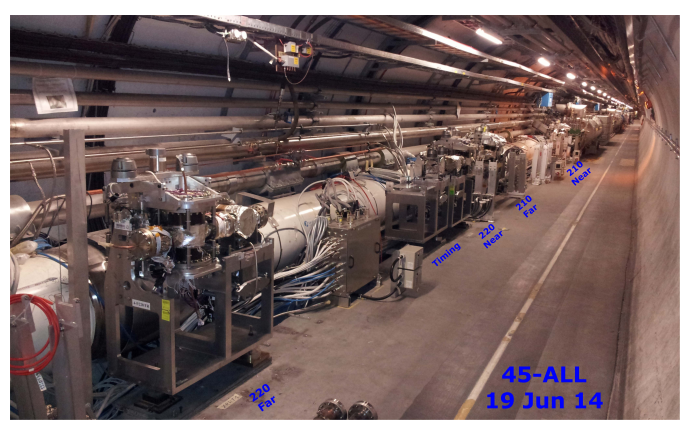

b)

Figure 1: The TOTEM experiment layout during the LHC Run1 and Run2. a) The position of the inelastic telescopes: T1 and T2, in the CMS experiment (top), and the first Roman Pots locations, along the LHC beam line, at $147 \mathrm{~m}$ (RP147) and $220 \mathrm{~m}$ (RP220) (bottom), during Run1. b) The new Roman Pot location along one tunnel arm during Run2. The $147 \mathrm{~m}$ stations are now installed at $210 \mathrm{~m}$, while the newly designed horizontal pots (Timing) are installed between the $220 \mathrm{~m}$ far and near.

proaches to determine the total cross-section, proving the reliability of the luminosity independent method. Measurements at $7 \mathrm{TeV}$, where double checked using different methods under the same beam conditions, while the ones at 8 and $13 \mathrm{TeV}$ where checked leveraging different beam and optics conditions. Moreover, with the data collected by the very forward telescopes T1 and T2, during special runs at $\sqrt{s}=7 \mathrm{TeV}$, also the double diffractive (DD) cross-section was measured in the pseudorapidity region $4.7<\left|\eta_{\min }\right|<6.5$ [13].

During the special runs, TOTEM provides minimum and zero bias triggers to the CMS experiment [15], making also possible physics studies in common between the two experiments, like the direct measurement of the forward pseudorapidity distributions [16]. The collaboration with CMS give birth to a join project: CMS-TOTEM Precision Proton Spectrometer (CT-PPS)[23], designed to take data during the LHC Run2 standard run with high luminosity. The project collected slightly less than $100 \mathrm{fb}^{-1}$, in the last two years of Run2 data taken, proving the feasibility of the project [26] that has been integrated into CMS as PPS subsystem, eventually.

\subsection{The TOTEM experiment}

The TOTEM experiment layout, during the LHC Run1 (2009-2013), is shown in Figure 1 along with the position of the inelastic telescopes, inside the CMS experiment, and Roman Pot stations, inside the tunnel [19]. The two inelastic telescopes consists of two arms located symmetrically on both sides of the LHC interaction point 5 (IP5): the T1 telescope with cathode strip chambers (CSC), placed at a distance of $9 \mathrm{~m}$ from IP5, covers the pseudorapidity range $3.1 \leq \eta \leq$ 4.7. The T2 telescope with gas electron multiplier (GEM) chambers, located at $\pm 13.5 \mathrm{~m}$ from IP5, covers the pseudorapidity range $5.3 \leq \eta \leq 6.5$. The pseudorapidity coverage of the two telescopes allowed the detection of $95 \%$ of the inelastic events, including events with diffractive mass down to $3.6 \mathrm{GeV}$.

The Roman Pot (RP) stations are located symmetrically on either side of the IP5 at distances of 215-220 m from it, inside the LHC tunnel. Each station is composed of two units (near and far, in the TOTEM jargon) separated by a distance of about $5 \mathrm{~m}$. A unit consists of 3 RPs, two 
approaching the outgoing beam vertically, from the top and the bottom, and one horizontally. During LHC Run1, each RP was equipped with a stack of 10 silicon strip detectors, designed with the specific objective of reducing the insensitive area at the edge facing the beam to only a few tens of micrometers. This design permits to measure the proton distance from the beam center, in both coordinates perpendicular to the beam, with a precision of about $11 \mu \mathrm{m}$ [18]. The movement and the alignment of all pots are monitored with a precision better than $20 \mu \mathrm{m}$ based on track reconstruction and external alignment tools. The large lever arm between the near and the far units allows the determination of the track angle in both projections with a precision of about $5 \mu \mathrm{rad}$. A more detailed description of the TOTEM detector and performance, in this configuration, can be found in $[19,20]$.

The experiment layout for the LHC Run2 (2015-2018), has been modified. New RP have been added and some of them relocated, as shown in Figure 1. In this updated configuration[21], the RP stations previously installed at $\pm 147 \mathrm{~m}$, from the interaction point, have been relocated at $\pm 210 \mathrm{~m}$, with one of the two unit, the far one, tilted around the beam axis by $8^{\circ}$. Moreover, two newly designed horizontal RP, have been installed between the two station at $\pm 220 \mathrm{~m}$. All RP have been refurbished and upgraded to stand the increase in luminosity foreseen. This new layout has been proposed to improve the apparatus performances, adding timing detectors, to resolve the pileup of multiple events in the same bunch crossing, and operate the experiment at higher luminosities. This program has been fulfilled by TOTEM, for the special runs with low luminosity, installing new timing detectors in a relocated vertical RP[22, 24, 25].

The RP upgrade was a feat that boosted the CT-PPS project. The CT-PPS collaboration installed, in two horizontal RP, new tracking detectors, based on the silicon pixels ones developed for the CMS tracker upgrade [23], while the diamond timing detectors, developed for the TOTEM upgrade, were installed in the new horizontal ones [24]. At the end, CT-PPS, was leveraging four different detector technologies: silicon strips and pixels, and diamond and Ultra Fast Silicon Detector (UFSD)[25], for tracking and timing respectively.

TOTEM will finish the cross section measurement campaign, during the LHC Run3 (20212023), when the machine will reach the maximum energy of $\sqrt{s}=14 \mathrm{TeV}$. For this measurement an upgrade of the T2 detector has been scheduled. The new T2 will fit in the reduced space left by the new LHC beamline configuration; it will be based on scintillator slabs readout by silicon photomultipliers (SiPM).

\subsection{Data set}

Measurements, reported in this contributions, are the result of the analysis of several data samples recorded, during LHC Run1 and Run2. In particular, during Run1, in June and October 2011, at $\sqrt{s}=7 \mathrm{TeV}$, July and October 2012, at $\sqrt{s}=8 \mathrm{TeV}$, and 2013 at $\sqrt{s}=2.76 \mathrm{TeV}$. Data have been recorded during special low intensity LHC fills with $\beta^{*}=3.5$ and $90 \mathrm{~m}$ and $\beta^{*}=90$ and $1000 \mathrm{~m}$, at energy of $7 \mathrm{TeV}$ and $8 \mathrm{TeV}$ respectively; while, for the $2.76 \mathrm{TeV}$ a $\beta^{*}=11 \mathrm{~m}$ was used. During Run2, two special runs have been performed during 2015 and 2016; both runs at the energy of $13 \mathrm{TeV}$ but with $\beta^{*}=90$ and $2500 \mathrm{~m}$ respectively.

In these special runs, the Roman Pots, are moved very close to the beam center, the actual distances depending on the transverse beam size value, $\sigma_{\text {beam }}$. Distances ranging from 3 to 10 times the $\sigma_{\text {beam }}$ as been set, depending by the beam optics and energies, and collimation scheme. 
The RP first-level trigger is based on track segment, in the near or the far unit. A coincidence between a proton on the left and the right side of the interaction point is requested in the elastic double-arm signature, in the vertical RP detectors, in either of the two diagonals (left top - right bottom or left bottom - right top). The RP detector was designed for the characteristics of a beam optics with large $\beta^{*}$. With this optics configuration most of the elastically scattered protons can be measured by the vertical RP. Elastically scattered protons cross the RP detectors close to the vertical plane, while diffractive protons are deviated to positive $x$-values in the vertical detectors. Defining in this way a nice topological criterion for selecting elastic candidate events [8]. Moreover a minimum bias trigger, requiring at least one track candidate in one of the T2 detector arm, was used during LHC Run1 special runs, making possible the direct measurement of the inelastic [5] and DD cross-section [13], along with the determination of the charged particle pseudorapidity density $[16,17]$.

Table 1: Summary of the cross-sections measured using a luminosity $(\mathscr{L})$ independent method. The full systematic uncertainty is reported for each measurements, see $[7,2,3,11]$ for a detailed description of this uncertainties.

\begin{tabular}{|l|c|c|c|c|}
\hline \multirow{2}{*}{} & \multicolumn{4}{|c|}{$\mathscr{L}$ independent using eq. 1.1 } \\
\cline { 2 - 5 } & $\sqrt{2.76} \mathrm{TeV}[14]$ (Prelim.) & $\sqrt{7} \mathrm{TeV}[2]$ & $\sqrt{8} \mathrm{TeV}[3]$ & $\sqrt{13} \mathrm{TeV}[11]$ \\
\hline$\sigma_{\text {tot }}(\mathrm{mb})$ & $84.7 \pm 3.3$ & $98.0 \pm 2.5$ & $101.7 \pm 2.9$ & $110.6 \pm 3.4$ \\
\hline$\sigma_{\text {inel }}(\mathrm{mb})$ & $62.8 \pm 2.9$ & $72.9 \pm 1.5$ & $74.7 \pm 1.7$ & $79.5 \pm 1.8$ \\
\hline$\sigma_{e l}(\mathrm{mb})$ & $21.8 \pm 1.4$ & $25.1 \pm 1.1$ & $27.1 \pm 1.4$ & $31.0 \pm 1.7$ \\
\hline
\end{tabular}

\section{Results}

The cross-sections obtained using the luminosity independent method of eq. 1.1, are reported in Table 1 and shown in Figure 2, along with the ones obtained by other experiments at LHC and using cosmic ray measurements (see [11] and references therein). In the table the total systematic uncertainty has been reported, obtained combining in quadrature all the systematic uncertainties, taking into account the correlations between contributions. For a more detailed description and discussion of the systematic uncertainties for these measurements see $[7,2,3,11]$ and references therein.

Given the various methods of measuring elastic and inelastic rates, it was possible to perform completely different estimation of the inelastic, elastic and total cross-sections, comparing the results for a better understanding of the systematic uncertainties and double checks the results.

In particular at $\sqrt{s}=7 \mathrm{TeV}$ the luminosity independent result [2] was shown to be consistent with the total cross section calculated by extrapolating the differential elastic cross-section to the optical point $|t|=0$, using Equation 1.2 [4, 7], as well as with a luminosity dependent method [6]. While from the direct measurement of the inelastic rates the inelastic cross-section was determined and compared with the one deduced from the difference between the total and the elastic cross section [5].

Also results at $\sqrt{s}=8 \mathrm{TeV}$ [3], has been double checked using the extrapolation of the differential elastic cross-section. This time, thanks to a very-high statistics collected at $\beta^{*}=90 \mathrm{~m}$, 


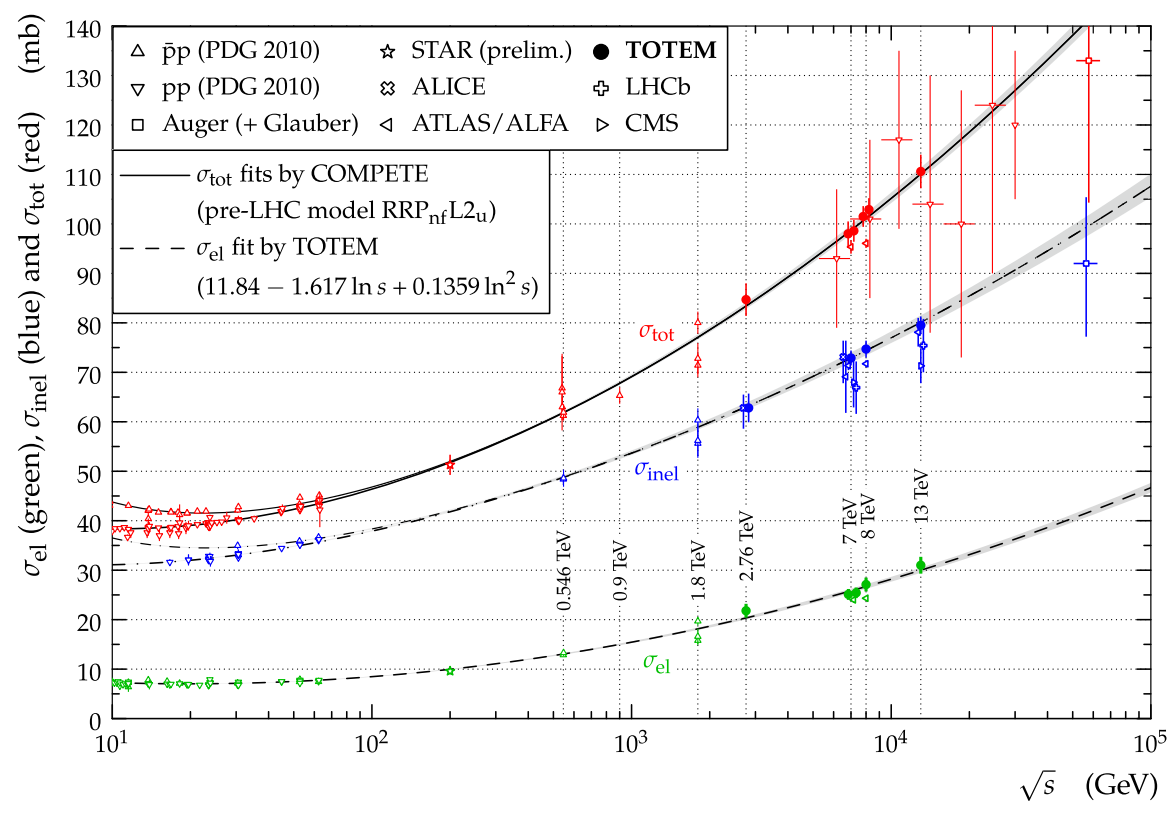

Figure 2: A compilation (see [11] and references therewith) of $p p$ and $\bar{p} p$ total $\left(\sigma_{t o t}\right)$, inelastic $\left(\sigma_{\text {inel }}\right)$ and elastic $\left(\sigma_{e l}\right)$ cross-sections measurements as a function of $\sqrt{s}$. The TOTEM measurements using the luminosity independent method are the full circle. The continuous black lines (lower for pp, upper for $\bar{p} \mathrm{p}$ ) represent the best fits of the total cross-section data by the COMPETE collaboration [28]. The dashed line results from a fit of the elastic cross-section data. The dash-dotted lines refer to the inelastic cross-section and are obtained as the difference between the continuous and dashed fits.

the TOTEM experiment was able to exclude a pure exponential behavior of the elastic differential cross-section [9], so refined parametrizations have been used for the extrapolation to the optical point. The significance of the exclusion is greater than $7 \sigma$ in the $|t|$ range from 0.027 to $0.2 \mathrm{GeV}^{2}$. Thanks to the data collected at this energy with $\beta^{*}=1000 \mathrm{~m}$, it was possible to explore the elastic scattering in the Coulomb Nuclear Interference (CNI) region. For the first time at LHC the $\rho$ parameter was measured, via the CNI, and was found to be $\rho=0.12 \pm 0.03$ [10].

The $\rho$ parameter has been measured also at $\sqrt{s}=13 \mathrm{TeV}$, thanks to the statistic collected in a special run with $\beta^{*}=2500 \mathrm{~m}$. Values obtained are: $\rho=0.09 \pm 0.01$ and $\rho=0.10 \pm 0.01$, depending on different physics assumptions and mathematical modelling [12]. This time the CNI region have been used to estimate the $\sigma_{t o t}$ using three different and independent approaches. This novel "Coulomb normalization" technique, was applied for the first time to LHC data, finding a results of $\sigma_{t o t}=110.3 \pm 3.5 \mathrm{mb}$, that combined with the luminosity independent method calculation yields to $\sigma_{t o t}=110.5 \pm 2.4 \mathrm{mb}$.

The unprecedented precision of the $\rho$ measurement, shown in Figure 3, combined with the TOTEM total cross-section measurements in an energy range from 2.76 to $13 \mathrm{TeV}$, has implied the exclusion of all the models classified and published by COMPETE. The $\rho$ results obtained by TOTEM are compatible with the predictions, from alternative theoretical models both in the Regge-like framework and in the QCD framework, of a colourless 3-gluon bound state exchange in the $t$-channel of the proton-proton elastic scattering. 


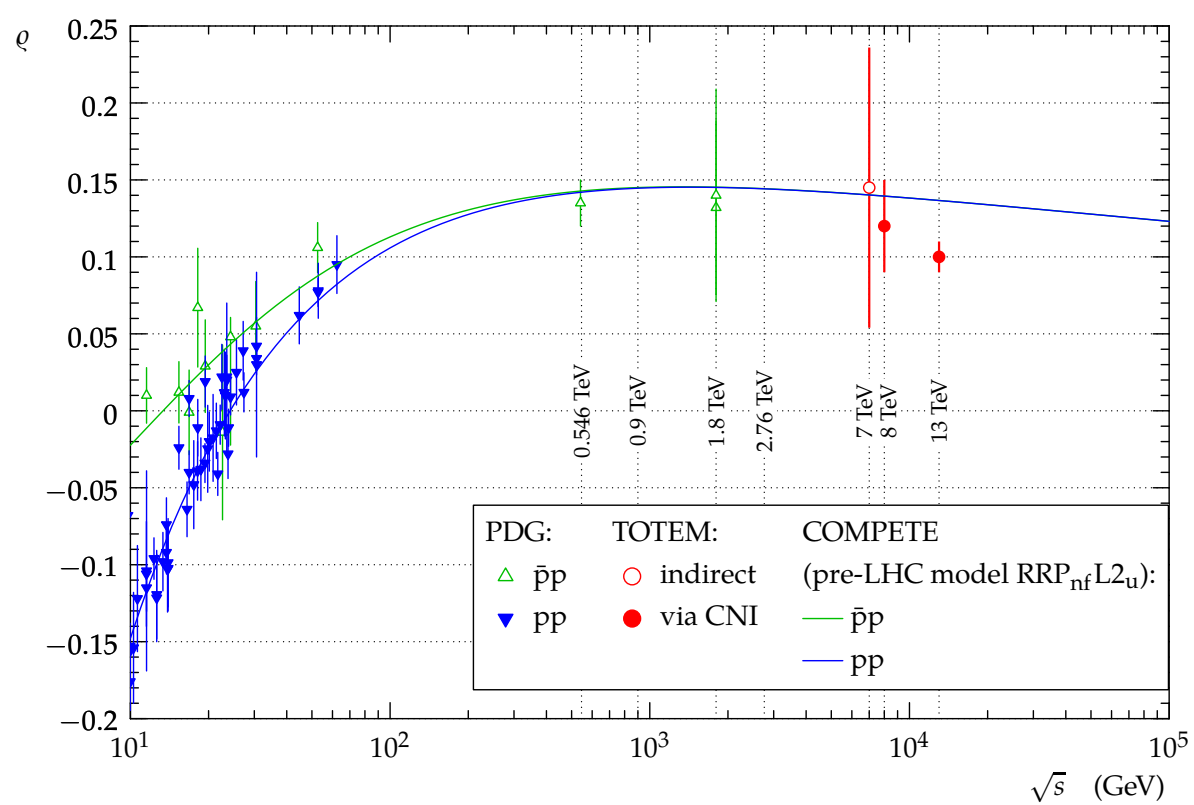

Figure 3: Dependence of the $\rho$ parameter on $\sqrt{s}$. The $p p$ (blue) and $p \bar{p}$ (green) data are taken from PDG [27]. TOTEM measurements $[10,12]$ are marked in red.

\section{Conclusions}

TOTEM has measured, for the first time at the LHC, the total, inelastic and elastic protonproton cross sections at $\sqrt{s}=2.76,7,8$ and $13 \mathrm{TeV}$ using a luminosity independent method. The method was validated comparing the elastic and inelastic cross sections measured in independent ways and using a novel "Coulomb normalization" technique based on QED data. Furthermore, for the first time at LHC, the $\rho$ parameter has been estimated studying the CNI region.

This measurement, combined with the total cross-section results, led to the exclusion of all the models classified and published by the COMPETE Collaboration, and shows a compatibility with predictions of a colorless 3 -gluon bound state exchange in the t-channel of proton-proton elastic scattering.

Acknowledgment:We are grateful to the beam optics development team for the design and the successful commissioning of the high $\beta^{*}$ optics and to the LHC machine coordinators for scheduling the dedicated fills.

This work was supported by the institutions listed on the front page and also by the Magnus Ehrnrooth foundation (Finland), the Waldemar von Frenckell foundation (Finland), the Academy of Finland, the Finnish Academy of Science and Letters (The Vilho, Yrjö and Kalle Väisälä Fund), the OTKA NK 101438 and the EFOP-3.6.1-16-2016-00001 grants (Hungary). Individuals have received support from Nylands nation vid Helsingfors universitet (Finland), from the MŠMT ČR (Czech Republic) and the János Bolyai Research Scholarship of the Hungarian Academy of Sciences and the NKP-17-4 New National Excellence Program of the Hungarian Ministry of Human Capacities.

\section{References}

[1] D. D‘Enterria et al., Astropart. Phys., 35 (2011) 98.

[2] G. Antchev et al. (TOTEM Collaboration), EPL, 101 (2013) 21004, doi:10.1209/0295-5075/101/21004. 
[3] G. Antchev et al., Phys. Rev. Lett. 111 (2013) 012001, doi: 10.1103/PhysRevLett.111.012001.

[4] G. Antchev et al. (TOTEM Collaboration), EPL, 101 (2013) 21002, doi:10.1209/0295-5075/101/21002.

[5] G. Antchev et al. (TOTEM Collaboration), EPL, 101 (2013) 21003, doi:10.1209/0295-5075/101/21003.

[6] G. Antchev et al. (TOTEM Collaboration), EPL, 96 (2011) 21002, doi: 10.1209/0295-5075/96/21002.

[7] G. Antchev et al. (TOTEM Collaboration), EPL, 95 (2011) 41001, doi: 10.1209/0295-5075/95/41001.

[8] G. Antchev et al. (TOTEM Collaboration), New J. Phys. 16 (2014) 103041, doi:10.1088/1367-2630/16/10/103041.

[9] G. Antchev et al. (TOTEM Collaboration), Nucl. Phys. B 899 (2015) 527, doi:10.1016/j.nuclphysb.2015.08.010.

[10] G. Antchev et al. (TOTEM Collaboration), Eur. Phys. J. C 76 (2016) 661, doi:10.1140/epjc/s10052-016-4399-8.

[11] G. Antchev et al. (TOTEM Collaboration), Eur. Phys. J. C, 79 (2019) 103, doi:10.1140/epjc/s10052-019-6567-0.

[12] G. Antchev et al. (TOTEM Collaboration), accepted for pubblication in Eur. Phys. J. C, arXiv:1812.04732.

[13] G. Antchev et al., Phys. Rev. Lett. , 111 (2013) 262001, doi: 10.1103/PhysRevLett.111.262001.

[14] F. Nemes (TOTEM Collaboration), PoS (DIS2017), 059 (2017).

[15] CMS Collaboration, JINST 3, S08004 (2008). doi:10.1088/1748-0221/3/08/S08004.

[16] The CMS and TOTEM Collaborations, EPJC 74 (2014) 3053, doi:10.1140/epjc/s10052-014-3053-6.

[17] G. Antchev et al. (TOTEM COllaboration), EPJC 75 (2015) 126, doi:10.1140/epjc/s10052-015-3343-7.

[18] E. Alagoz et al., JINST, 8, P06009 (2013), doi:10.1088/1748-0221/8/06/P06009.

[19] G. Anelli et al. (TOTEM Collaboration), JINST, 3, S08007 (2008), doi:10.1088/1748-0221/3/08/S08007.

[20] G. Antchev et al., Int. J. Mod. Phys. A 28, 1330046 (2013), doi: 10.1142/S0217751X13300469.

[21] TOTEM Collaboration, TOTEM Upgrade Proposal, CERN-LHCC-2013-009, (2013), url: https://cds.cern.ch/record/1554299.

[22] TOTEM Collaboration, Timing Measurements in the Vertical Roman Pots of the TOTEM Experiment, CERN-LHCC-2014-020, (2014), url: https://cds.cern.ch/record/1753189 .

[23] M. Albrow et al. (CMS-TOTEM Collaboration), CMS-TOTEM Precision Proton Spectrometer, CERN-LHCC-2014-021, (2014), url: https://cds.cern.ch/record/1753795 .

[24] G. Antchev et al., JINST, 12, P03007 (2017), doi:10.1088/1748-0221/12/03/P03007.

[25] M. Berretti et al., JINST, 12, P03024 (2017), doi:10.1088/1748-0221/12/03/P03024.

[26] A. M. Sirunyan, A. Tumasyan et al., J. High Energ. Phys. , 2018, 153 (2018), doi:10.1007/JHEP07(2018)153.

[27] C. Patrignani et al., (Particle Data Group), Chin. Phys., C40, 100001 (2016).

[28] J. R. Cudell et al. (COMPETE Collaboration), Phys. Rev. Lett. 89, 201801 (2002). 